\title{
1 \\ Basic Concepts
}

\subsection{Introduction}

A brief glance through almost any recently published medical journal will show that statistical methods are playing an increasingly visible role in modern medical research. At the very least, most research papers quote (at least) one 'p-value' to underscore the 'significance' of the results which the authors wish to communicate. At the same time, a growing number of papers are now presenting the results of relatively sophisticated, 'multi-factor' statistical analyses of complex sets of medical data. This proliferation in the use of statistical methods has also been paralleled by the increased involvement of professionally trained statisticians in medical research as consultants to and collaborators with the medical researchers themselves.

The primary purpose of this book is to provide medical researchers with sufficient understanding to enable them to read, intelligently, statistical methods and discussion appearing in medical journals. At the same time, we have tried to provide the means for researchers to undertake the simpler analyses on their own, if this is their wish. And by presenting statistics from this perspective, we hope to extend and improve the common base of understanding which is necessary whenever medical researchers and statisticians interact.

It seems obvious to us that statisticians involved in medical research need to have some understanding of the related medical knowledge. We also believe that in order to benefit from statistical advice, medical researchers require some understanding of the subject of statistics. This first chapter provides a brief introduction to some of the terms and symbols which recur throughout the book. It also establishes what statisticians talk about (random variables, 
probability distributions) and how they talk about these concepts (standard notation). We are very aware that this material is difficult to motivate; it seems so distant from the core and purpose of medical statistics. Nevertheless, 'these dry bones' provide a skeleton which allows the rest of the book to be more precise about statistics and medical research than would otherwise be possible. Therefore, we urge the reader to forbear with these beginnings, and read beyond the end of chapter 1 to see whether we do not put flesh onto these dry bones.

\subsection{Random Variables, Probability Distributions and Some Standard Notation}

Most statistical work is based on the concept of a random variable. This is a quantity that, theoretically, may assume a wide variety of actual values, although in any particular realization we only observe a single value. Measurements are common examples of random variables; take the weights of individuals belonging to a well-defined group of patients, for example. Regardless of the characteristic that determines membership in the group, the actual weight of each individual patient is almost certain to differ from that of other group members. Thus, a statistician might refer to the random variable representing the weight of individual patients in the group, or population of interest. Another example of a random variable might be a person's systolic blood pressure; the variation in this measurement from individual to individual is frequently quite substantial.

To represent a particular random variable, statisticians generally use an upper case Roman letter, say X or Y. The particular value which this random variable represents in a specific case is often denoted by the corresponding lower case Roman letter, say x or y. The probability distribution (usually shortened to the distribution) of any random variable can be thought of as a specification of all possible numerical values of the random variable, together with an indication of the frequency with which each numerical value occurs in the population of interest.

It is common statistical shorthand to use subscripted letters $-\mathrm{x}_{1}, \mathrm{x}_{2}, \ldots$, $\mathrm{x}_{\mathrm{n}}$, for example - to specify a set of observed values of the random variable $\mathrm{X}$. The corresponding notation for the set of random variables is $X_{i}, i=1,2, \ldots, n$, where $X_{i}$ indicates that the random variable of interest is labelled $X$ and the symbols $i=1,2, \ldots, n$ specify the possible values of the subscripts on X. Similarly, using $\mathrm{n}$ as the final subscript in the set simply indicates that the size of the set may vary from one instance to another, but in each particular instance it will be a fixed number. 
Subscripted letters constitute extremely useful notation for the statistician, who must specify precise formulae which will subsequently be applied in particular situations which vary enormously. At this point it is also convenient to introduce the use of $\Sigma$, the upper case Greek letter sigma. In mathematics, $\Sigma$ represents summation. To specify the sum $\mathrm{X}_{1}+\mathrm{X}_{2}+\mathrm{X}_{3}$ we would simply write $\sum_{i=1}^{3} \mathrm{X}_{\mathrm{i}}$. This expression specifies that the subscript i should take the values 1,2 and 3 in turn, and we should sum the resulting variables. For a fixed but unspecified number of variables, say $n$, the sum $X_{1}+X_{2}+\ldots+X_{n}$ would be represented by $\sum_{i=1}^{n} X_{i}$.

A set of values $\mathrm{x}_{1}, \mathrm{x}_{2}, \ldots, \mathrm{x}_{\mathrm{n}}$ is called a sample from the population of all possible occurrences of $\mathrm{X}$. In general, statistical procedures which use such a sample assume that it is a random sample from the population. The random sample assumption is imposed to ensure that the characteristics of the sample reflect those of the entire population, of which the sample is often only a small part.

There are two types of random variables. If we ignore certain technicalities, a discrete random variable is commonly defined as one for which we can write down all its possible values and their corresponding frequencies of occurrence. In contrast, continuous random variables are measured on an interval scale, and the variable can assume any value on the scale. Of course, the instruments which we use to measure experimental quantities (e.g., blood pressure, acid concentration, weight, height, etc.) have a finite resolution, but it is convenient to suppose, in such situations, that this limitation does not prevent us from observing any plausible measurement. Furthermore, the notation which statisticians have adopted to represent all possible values belonging to a given interval is to enclose the end-points of the interval in parentheses. Thus, $(a, b)$ specifies the set of all possible values between a and $b$, and the symbolic statement ' $\mathrm{a}<\mathrm{X}<\mathrm{b}$ ' means that the random variable $\mathrm{X}$ takes a value in the interval specified by $(a, b)$.

The probability distribution of a random variable is often illustrated by means of a histogram or bar graph. This is a picture which indicates how frequently each value of the random variable occurs, either in a sample or in the corresponding population. If the random variable is discrete, the picture is generally a simple one to draw and to understand. Figure 1.1a shows a histogram for the random variable, $S$, which represents the sum of the showing faces of two fair dice. Notice that there are exactly 11 possible values for S. In contrast to this situation, the histogram for a continuous random variable, say systolic blood pressure, $\mathrm{X}$, is somewhat more difficult to draw and to understand. One such histogram is presented in figure 1.1b. Since the picture is intended to show both the possible values of $\mathrm{X}$ and also the frequency with which they arise, each rectangular block in the graph has an area equal to the propor- 


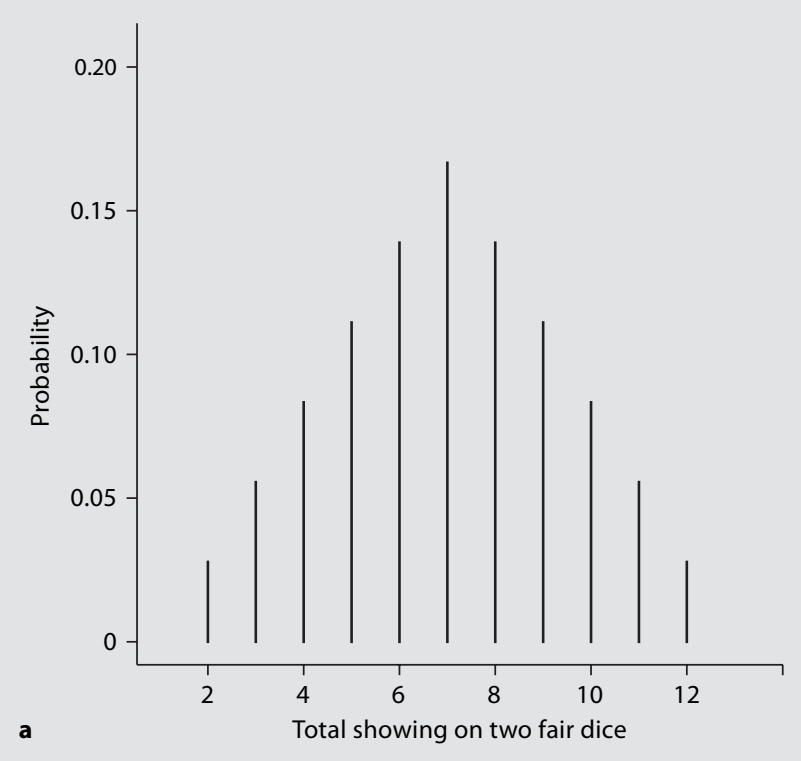
Total showing on two fair dice

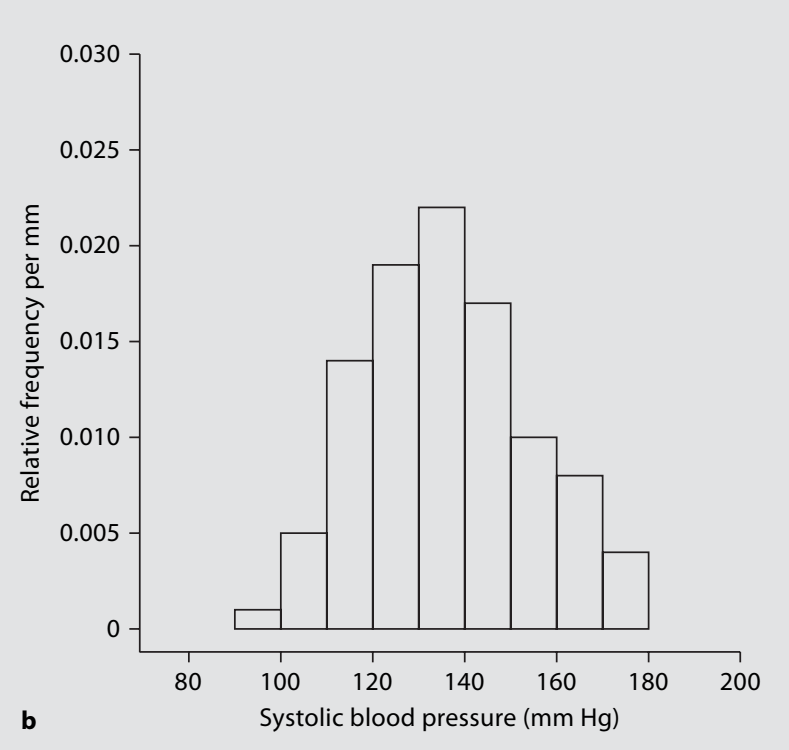

Fig. 1.1. Histograms of random variables. a The discrete random variable, S, representing the sum of the showing faces for two fair dice. $\mathbf{b}$ One hundred observations on the continuous random variable, $\mathrm{X}$, representing systolic blood pressure. 
tion of the sample represented by all outcomes belonging to the interval on the base of the block. This has the effect of equating frequency, or probability of occurrence, with area and is known as the 'area = probability' equation for continuous random variables.

To a statistician, histograms are simply an approximate picture of the mathematical way of describing the distribution of a continuous random variable. A more accurate representation of the distribution is obtained by using the equation of a curve which can best be thought of as a 'smooth histogram'; such a curve is called a probability density function. A more convenient term, and one which we intend to use, is probability curve.

Figure 1.2a shows the probability curve, or smooth histogram, for the continuous random variable, $\mathrm{X}$, which we used above to represent systolic blood pressure. This curve is, in fact, the probability curve which has the characteristic shape and equation known as a 'normal distribution'. Random variables that have a normal distribution will recur in subsequent chapters, and we intend to explain their properties and uses in more detail at that time. For the present, however, we want to concentrate on the concept of the area $=$ probability equation. Figure 1.2b shows two shaded areas. One is the area below the curve and above the interval $(110,130)$. Recall that the symbol $(110,130)$ represents all blood pressure measurements between 110 and $130 \mathrm{~mm} \mathrm{Hg}$. Because of the area $=$ probability equation for the continuous random variable $\mathrm{X}$, the shaded area above $(110,130)$ corresponds, pictorially, to the probability that systolic blood pressure in the population is between 110 and $130 \mathrm{~mm} \mathrm{Hg}$. This area can be calculated mathematically, and in this particular example the value is 0.323 . To represent this calculation in a symbolic statement we would write $\operatorname{Pr}(110<\mathrm{X}<130)=0.323$; the equation states that the probability that $\mathrm{X}$, a systolic blood pressure measurement in the population, is between 110 and $130 \mathrm{~mm} \mathrm{Hg}$ is equal to 0.323 .

The second shaded area in figure $1.2 \mathrm{~b}$ is the area below the probability curve corresponding to values of X in the interval $(165, \infty)$, i.e., the probability that a systolic blood pressure measurement in the population exceeds $165 \mathrm{~mm} \mathrm{Hg}$. By means of certain calculations we can determine that, for this specific example, the probability that systolic blood pressure exceeds $165 \mathrm{~mm}$ $\mathrm{Hg}$ is 0.023 ; the concise mathematical description of this calculation is simply $\operatorname{Pr}(\mathrm{X}>165)=0.023$.

Although the probability curve makes it easy to picture the equality of area and probability, it is of little direct use for actually calculating probabilities since areas cannot be read directly from a picture or sketch. Instead, we need a related function called the cumulative probability curve. Figure 1.3 presents the cumulative probability curve for the normal distribution shown in figure 1.2a. The horizontal axis represents the possible values of the random 

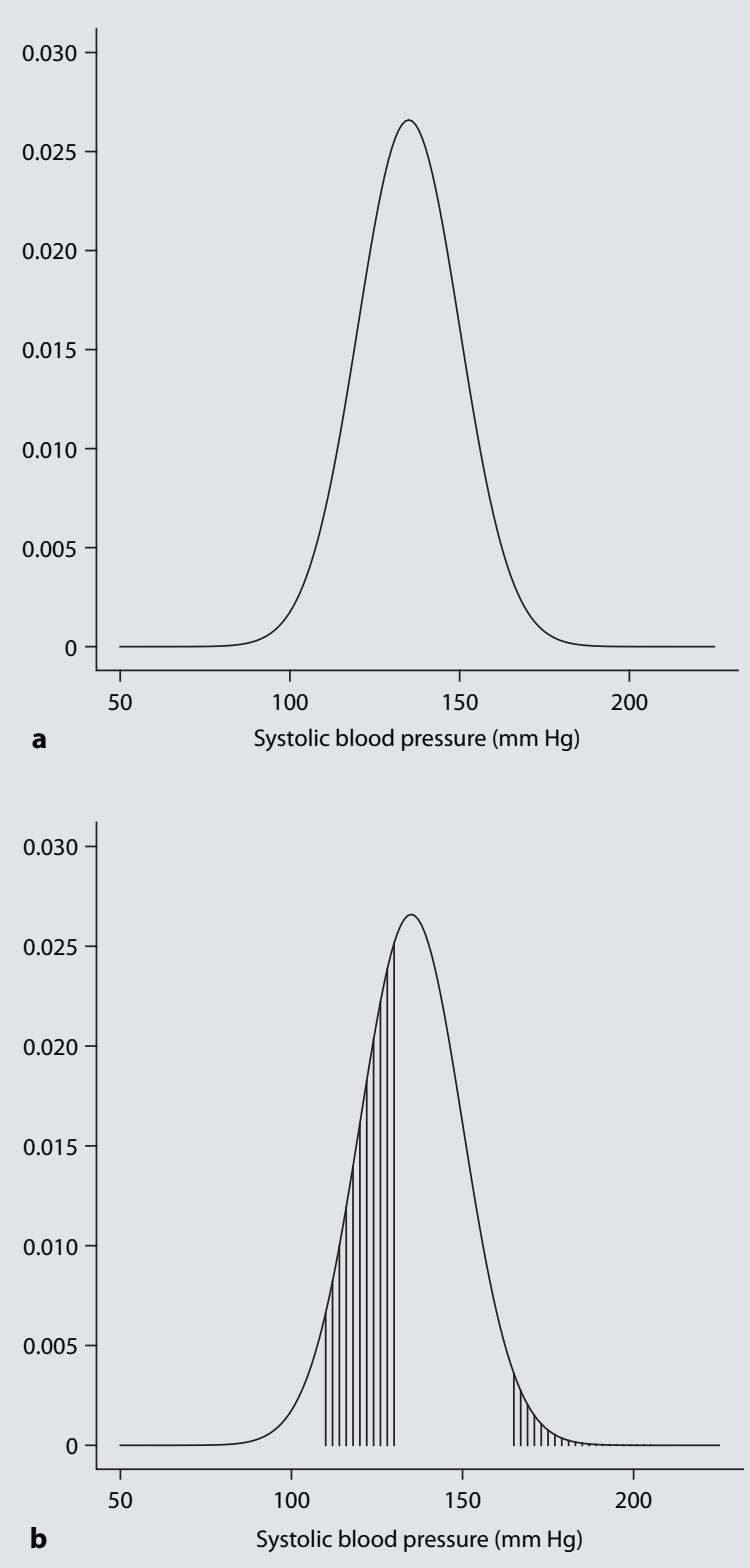

Fig. 1.2. A probability curve for the continuous random variable, $X$, representing systolic blood pressure. a As a smooth histogram. b With shaded areas corresponding to $\operatorname{Pr}(110<\mathrm{X}<130)$ and $\operatorname{Pr}(\mathrm{X}>165)$. 


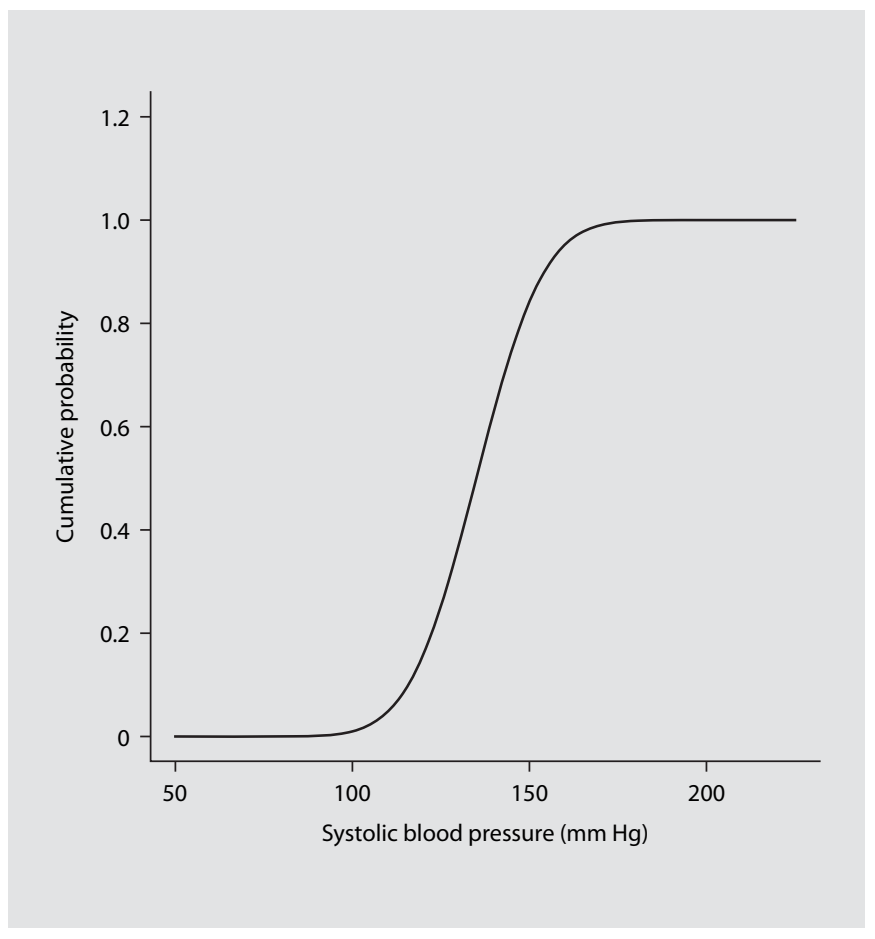

Fig. 1.3. The cumulative probability curve for the random variable, $X$, representing systolic blood pressure.

variable X; the vertical axis is a probability scale with values ranging from zero to one. The cumulative probability curve specifies, for each value a, say, on the horizontal axis, the probability that the random variable $\mathrm{X}$ takes a value which is at most a, i.e., $\operatorname{Pr}(X \leq \mathrm{a})$. This probability is precisely the area below the probability curve corresponding to values of $\mathrm{X}$ in the interval $(-\infty, a)$. In particular, if $\mathrm{a}=\infty$, i.e., $\operatorname{Pr}(-\infty<\mathrm{X}<\infty)$, the value of the cumulative probability curve is one, indicating that $\mathrm{X}$ is certain to assume a value in the interval $(-\infty$, $\infty)$. In fact, this result is a necessary property of all cumulative probability curves, and is equivalent to the statement that the area under any probability curve is always equal to one.

Clearly, a cumulative probability curve is more useful than the corresponding probability curve for actually calculating probabilities. For example, since the area under the probability curve always equals one, it follows that

$$
\operatorname{Pr}(\mathrm{X}>\mathrm{a})=1-\operatorname{Pr}(\mathrm{X} \leq \mathrm{a}) .
$$


Thus, the cumulative probability curve can be used to calculate the probability corresponding to the interval $(a, \infty)$. And for the interval $(a, b)$, where a and $\mathrm{b}$ are two specific values, it is fairly easy to show that

$$
\operatorname{Pr}(\mathrm{a}<\mathrm{X}<\mathrm{b})=\operatorname{Pr}(\mathrm{X}<\mathrm{b})-\operatorname{Pr}(\mathrm{X} \leq \mathrm{a}) ;
$$

this is simply a difference of the cumulative probability curve evaluated at the two points $a$ and $b$.

\section{Comment:}

Most readers have probably noticed that $\$ 1.2$ did not contain any specific formulae for calculating probabilities, particularly in the case of continuous random variables. The reason for this is simple. More frequently than not, the calculations are sufficiently formidable, numerically, that statisticians have prepared standardized tables to make the evaluation of probabilities relatively simple. These tables, which often are tabulations of the cumulative probability curve, are generally called statistical tables; the values which we quoted for the normal distribution shown in figure $1.2 \mathrm{~b}$ were obtained from a statistical table for the normal distribution. In subsequent chapters, as various common probability distributions arise in the exposition, we will discuss how to use the relevant statistical table, or the results of probability calculations provided by a statistical software package.

\subsection{Characteristics of a Distribution: Mean, Median and Variance}

If we wish to be absolutely precise about the distribution of a certain random variable, say $\mathrm{X}$, then we must specify the equation of the probability curve if it is a continuous random variable, or the values of $X$ and the probability with which they occur if $\mathrm{X}$ is a discrete random variable. However, if we only wish to indicate something about the distribution of $\mathrm{X}$ in general terms, it often suffices to specify the location and spread of the distribution of X. In common, everyday terms this is equivalent to the two usual answers to the question 'Where is X's office?'. If you wish to be precise you would answer 'X's office is Room 703 at 1024 Columbia Street, in the Cabrini Tower'. On the other hand, if you were only planning to offer a general answer to the question you would reply 'X's office is on Capitol Hill'.

\subsubsection{Location}

A measure of location for the distribution of a random variable, $\mathrm{X}$, should tell us the value which is roughly central, in some sense, to the range of values where $\mathrm{X}$ is regularly observed to occur. The most common measure of location 


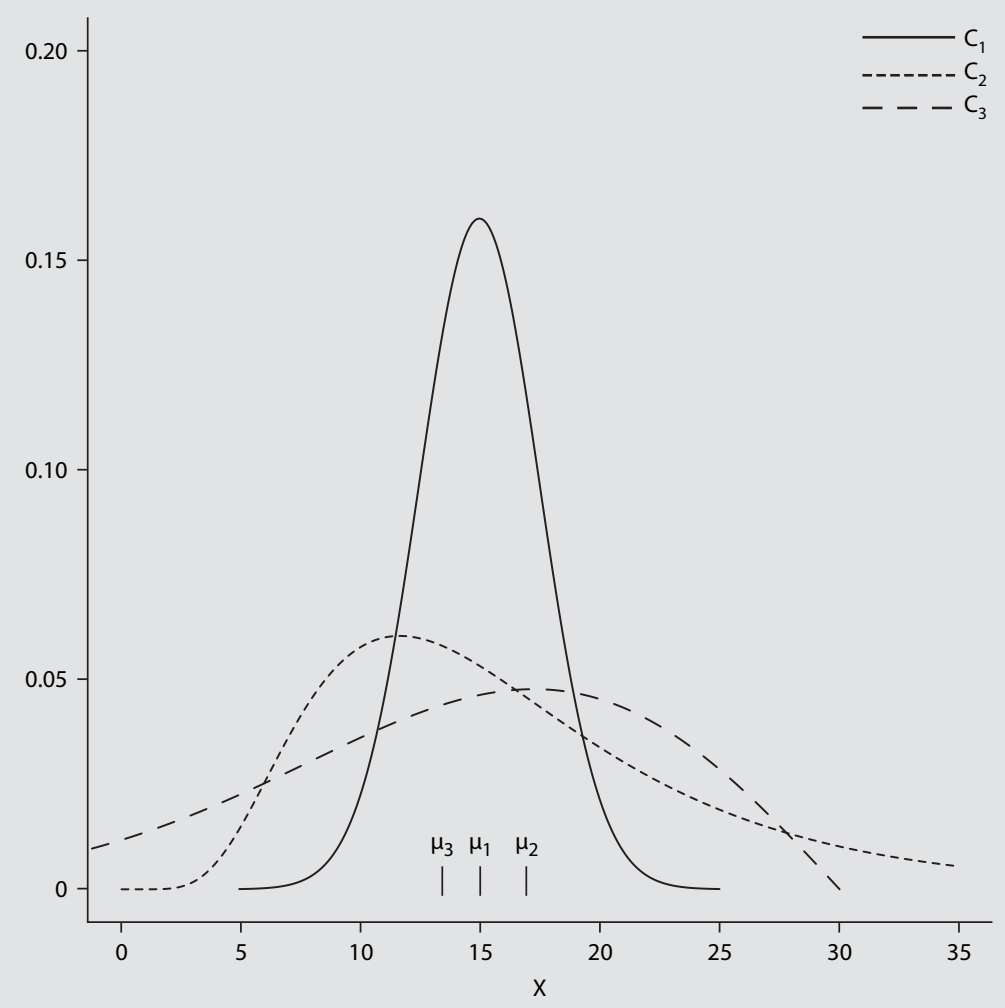

Fig. 1.4. Three probability curves, $C_{1}, C_{2}$ and $C_{3}$, having the same median (15) but different means $\mu_{1}, \mu_{2}$ and $\mu_{3}$, respectively.

used by statisticians is the mean or expected value of $\mathrm{X}$; this is denoted by the symbol $\mathrm{E}(\mathrm{X})$. If we think, for a moment, of a probability associated with the distribution of $\mathrm{X}$ as mass, then the mean value, $\mathrm{E}(\mathrm{X})$, is located at the center of mass. The Greek letter $\mu$ is often used to represent $\mathrm{E}(\mathrm{X})$.

A second measure of location is the median value of $\mathrm{X}$. Most readers will recognize the median as the measure of location which is commonly used in the medical literature. The median of $\mathrm{X}$ is that particular value which equally divides the probability in the distribution of $\mathrm{X}$, i.e., with probability $1 / 2$ an observed value of $\mathrm{X}$ will exceed the median and with probability $1 / 2$ an observed value will not exceed the median.

In general, the median of $\mathrm{X}$ and the mean, $\mathrm{E}(\mathrm{X})$, are different because the median is less influenced by extreme values of $\mathrm{X}$ which might occur than is 
$\mathrm{E}(\mathrm{X})$. However, if the distribution of $\mathrm{X}$ below the median is a mirror image of the upper half of the distribution, then the distribution is said to be symmetric and the median and mean will coincide. To illustrate some of the differences between the mean and the median, figure 1.4 shows three probability curves which have the same median but different means.

\subsubsection{Spread}

The dispersion or spread of a distribution indicates how variable the quantity represented by $\mathrm{X}$ is expected to be from one observation to the next. The most common measure of spread for a random variable, $\mathrm{X}$, is called the variance of $\mathrm{X}$.

To define variance we begin with the constructed variable $(X-\mu)$, where $\mu=\mathrm{E}(\mathrm{X})$; recall that $\mathrm{E}(\mathrm{X})$ is the mean of $\mathrm{X}$ and indicates, roughly, the center of the distribution. The constructed variable $(X-\mu)$ measures both the direction and the amount by which $\mathrm{X}$ deviates from its mean value $\mu$. For the purposes of measuring spread in the distribution of $\mathrm{X}$, the direction of this deviation is of less importance than the magnitude; however, large deviations in either direction influence the spread of the distribution more than small deviations. Therefore, we use $(X-\mu)^{2}$, the square of our constructed random variable, to indicate spread and call $\mathrm{E}\left\{(\mathrm{X}-\mu)^{2}\right\}$, the expected value of this random variable, the variance of the distribution of $\mathrm{X}$. For convenience, the variance is frequently represented by the Greek symbol $\sigma^{2}$.

The square root of the variance, which is usually indicated by the symbol $\sigma$, is called the standard deviation of the distribution of X. By specifying the mean, $\mu$, and standard deviation, $\sigma$, for the distribution of $X$, we are providing an approximate or general description of the entire distribution. But to evaluate probabilities for a distribution, or to perform other calculations, we need more specific information than just the mean and standard deviation. For accurate calculations we also require the equation of the cumulative probability curve or suitable tables. However, these are details which we intend to discuss in later chapters.

Too often the beginnings of any subject will kill the enthusiasm of the most eager reader. However, now that the groundwork has been done, we can proceed to discuss some of the basic procedures which statisticians have developed. The first of these which we will consider, and therefore the subject of chapter 2 , is called a test of significance. 
